ISSN 2179-6750

\title{
A atuação do cirurgião-dentista, vinculado a um programa de residência multiprofissional em saúde, no combate à COVID-19 na Atenção Primária à Saúde: relato de experiência
}

\author{
The dentist's performance, linked to a Multiprofessional Residency \\ Program in Health, in the fight against COVID - 19 \\ in Primary Health Care: experience report
}

La actuación del cirujano-dentista, vinculado a un Programa de Residencia Multiprofesional en Salud, en el combate a la COVID-19 en la Atención

Primaria de Salud: informe de experiencia

\author{
Jamille Souza Xavier dos SANTOS ${ }^{(1)}$ \\ Andresa Santos SILVA ${ }^{(1)}$ \\ Leilane dos Anjos de CARVALHO(1) \\ Juliana Oliveira SOARES(1) \\ Síntique Priscila Alves LOPES(1) \\ Marcela Beatriz Aguiar MOREIRA ${ }^{(1)}$
}

Recebido: 17 maio 2020

Revisado: 03 jun 2020

Aceito: 01 jul 2020

Autor de correspondência: Síntique Priscila Alves Lopes alvessintique@gmail.com

Conflito de interesses: Os autores declaram não haver nenhum interesse profissional ou pessoal que possa gerar conflito de interesses em relação a este manuscrito.
(1)Fundação Estatal Saúde da Família FESF-SUS, Programa de Residência Multiprofissional em Saúde da Família, Camaçari, BA, Brasil.

\section{Resumo}

Identificado no final do ano de 2019, em uma cidade da China, o SARS-coV-2, agente da doença COVID-19, provocou um estado de pandemia e alerta global. No Brasil, o setor saúde transformou a dinâmica do seu processo de trabalho, inclusive da Atenção Primária à Saúde. Dentre os profissionais de saúde atuantes nesse nível de atenção, é fundamental destacar a essencialidade do cirurgião-dentista como ator no enfrentamento à COVID-19. Diante desse contexto, o objetivo do presente artigo é relatar a atuação de cirurgiões-dentistas, vinculados a um Programa de Residência Multiprofissional em Saúde, em uma Unidade de Saúde da Família, alocada em um município do nordeste brasileiro, no cenário da pandemia relativa à COVID-19. Trata-se de um relato de experiência de caráter descritivo e exploratório, desenvolvido pela Equipe de residentes do Núcleo de Odontologia, sob supervisão da preceptoria e apoio pedagógico vinculados ao núcleo profissional, no período entre março e junho de 2020. Para tanto, a trajetória metodológica percorrida apoiou-se na análise retrospectiva de estudos/normativas que tiveram enfoque na temática abordada, contribuindo para o processo de discussão e reflexão das experiências vivenciadas no contexto sanitário vigente. A partir da construção, em equipe, de um novo fluxo de atendimento na unidade de saúde, durante este período, gerou reflexões magníficas e que mereciam ser compartilhadas. Entrementes, identificou-se que esta categoria profissional se destacou no aprimoramento de habilidades de cuidado que extrapolam a cavidade bucal, principalmente no que tange ao acolhimento à demanda espontânea.

Descritores: Atenção Primária à Saúde; Equipe de Assistência ao Paciente; Odontólogos; Internato e Residência; Infecções por Coronavirus. 


\section{Abstract}

Identified at the end of 2019 in a Chinese city, SARS-coV-2, the agent of COVID-19 disease, caused a pandemic state and global alert. In Brazil, the health sector has been involved in transforming the entire dynamics of its work process, including Primary Health Care. It is fundamental to emphasize the importance of the multiprofessional team's action in the fight against COVID-19, emphasizing the essentiality of the dentist as an actor in this fight. In this context, the objective of the present article is to report the performance of dentists, linked to a Multiprofessional Health Residency Program, in a Family Health Unit, allocated in one town northeastern Braziliana, in the scenario of the pandemic related to COVID-19. It is a descriptive and exploratory experience report, developed by the Residents' Team of the Dentistry Nucleus, under the supervision of the preceptorate linked to the professional nucleus, in the period between March and June 2020. The methodological trajectory was based on a retrospective analysis of studies and standards that focused on the topic addressed, contributing to the process of discussion and reflection on the experiences lived in the current health context. In addition, the team building of a new service flow during this period generated magnificent reflections that deserved to be shared. Meanwhile, it was identified that this category stood out in the improvement of care skills that go beyond the oral cavity, especially when it comes to welcoming spontaneous demand.

Keywords: Primary Health Care; Patient Care Team; Dentists; Internship and Residence; Coronavirus Infections.

\section{Resumen}

Identificado a finales de 2019 en una ciudad china, SARS-coV-2, el agente de la enfermedad COVID19, causó un estado de pandemia y alerta mundial. En el Brasil, el sector de la salud ha participado en la transformación de toda la dinámica de su proceso de trabajo, incluida la atención primaria de la salud. Es fundamental destacar la importancia de la acción del equipo multiprofesional en la lucha contra COVID-19, haciendo hincapié en la esencialidad del dentista como actor en esta lucha. En este contexto, el objetivo del presente artículo es informar sobre el desempeño de los dentistas, vinculados a un Programa Multiprofesional de Residencia Sanitaria, en una Unidad de Salud Familiar, asignada en una ciudad noreste del Brasil, en el escenario de la pandemia relacionada con el COVID19. Se trata de un informe descriptivo y exploratorio de la experiencia, elaborado por el Equipo de Residentes del Núcleo de Odontología, bajo la supervisión del preceptorado vinculado al núcleo profesional, en el período comprendido entre marzo y junio de 2020. La trayectoria metodológica se basó en un análisis retrospectivo de los estudios y normas que se centraron en el tema tratado, contribuyendo al proceso de debate y reflexión sobre las experiencias vividas en el contexto sanitario actual. Además, la creación de un nuevo flujo de servicio durante este período generó magníficas reflexiones que merecían ser compartidas. Mientras tanto, se identificó que esta categoría se destacaba en la mejora de las habilidades de cuidado que van más allá de la cavidad oral, especialmente cuando se trata de acoger la demanda espontánea.

Palabras-claves: Atención Primaria de Salud; Grupo de Atención al Paciente; Odontólogos; Internado y Residencia; Infecciones por Coronavirus.

\section{Introdução}

Diante do cenário emergencial provocado pela pandemia relativa à Doença Coronavírus 2019 - COVID-19, foi percebido que o setor saúde não estava preparado para o impacto promovido por esta enfermidade, principalmente no que se refere à disponibilidade de recursos humanos e materiais, como Equipamentos de Proteção Individual - EPI e respiradores mecânicos. Neste sentido, os obstáculos prosseguiram também pelo setor da economia, em detrimento à necessidade de distanciamento social, visto que a possibilidade de contágio por contato, através de tosse, espirro ou fala, tornouse constante. Assim, constata-se que o cirurgião-dentista (CD) aponta como categoria de 
risco potencial para contaminação, tendo em vista que seu processo de trabalho envolve justamente a cavidade oral, rica em fluido salivar, o qual, rotineiramente, é reconhecido como algo que auxilia na prevenção de doenças bucais, mas que, por outro lado, pode favorecer a transmissão de doenças virais.

Para compreender esse novo contexto laboral da Odontologia, diante do cenário supracitado, se faz iminente o destaque acerca de aspectos biológicos da COVID-19, a qual é caracterizada como doença respiratória aguda causada pelo SARS-CoV-2 (Severe Acute Respiratory Syndrome Coronavirus 2). Coronavírus é uma família de vírus que têm conformação em um núcleo genético envolvido por picos de proteínas, as quais estão distribuídas no envelope viral permitindo aparência de coroa, por isso a denominação "corona". Os hospedeiros comuns destes vírus são animais como camelos, gatos e morcegos. No entanto, alguns integrantes dessa família são capazes de infectar humanos, a exemplo do MERS-CoV, SARS-CoV e o atual, SARS CoV-2, agente etiológico da COVID-19., ${ }^{12}$

Este novo coronavírus foi identificado pela primeira vez em dezembro do ano de 2019, em Wuhan, na China. Sabe-se que a via de transmissão ocorre, principalmente, por gotículas respiratórias de pessoas sintomáticas para outras pessoas que estão em contato próximo, pelo contato direto com a pessoa infectada, ou por contato com objetos e superfícies contaminados. Para as infecções confirmadas por SARS CoV-2, os pacientes têm apresentado sintomas que envolvem o trato respiratório, gastrointestinal, musculoesquelético e neurológico, apresentando comumente febre (83-98\%), tosse (76$82 \%$ ) e dispnéia (31-55\%). Nos quadros que evoluem para a gravidade, o paciente pode ser acometido por pneumonia podendo haver progressão para morte ${ }^{3}$.

No Brasil, o Sistema Único de Saúde - SUS, através do Ministério da Saúde, orientou os cuidados nos diversos níveis de atenção, de maneira que a atenção básica se manteve como porta de entrada do sistema. Desta forma, as Unidades de Saúde da Família - USF e Unidades Básicas de Saúde - UBS foram conduzidas à implantação do seguinte fluxo para acolhimento e favorecimento do diagnóstico clínico-epidemiológico de usuários: realização de anamnese criteriosa, verificação de contato recente deste com alguma pessoa diagnosticada com a doença, ou tenha feito viagem para lugares com elevado número de casos confirmados. Em caso de resposta positiva para algum destes questionamentos, além de considerar a apresentação dos sintomas respiratórios supracitados comuns da doença (descartando sinais de gravidade), orienta-se a entrega de máscara cirúrgica e isolamento domiciliar por 14 dias. Posteriormente, a equipe de saúde que acolheu este usuário deverá realizar a notificação da doença para a vigilância epidemiológica e posterior acompanhamento do caso. ${ }^{4}$ 
É fundamental salientar a importância da atuação da equipe multiprofissional frente à resolutividade de casos sintomáticos leves e encaminhamento correto dos casos graves. O fluxo de atendimento para pacientes sintomáticos, segundo o Ministério da Saúde, envolve a identificação precoce do paciente com sintomas de síndrome gripal, conforme citado acima, e a realização do atendimento em uma sala ou área isolada, com janelas abertas e ar condicionado desligado, com o intuito de evitar a circulação desse usuário pela unidade de saúde. A presença de profissionais de saúde, como agentes comunitários de saúde (ACS), técnicos de enfermagem, profissionais do Núcleo Ampliado de Saúde da Família e Atenção Básica - NASF-AB, cirurgiões-dentistas, enfermeiros e médicos, nessas ações é imprescindível para agilizar o atendimento e direcionar o tratamento. ${ }^{5}$ Vale ressaltar que os ACS são fundamentais na comunicação com os usuários e suas famílias por meio de tecnologias à distância, principalmente àqueles que estejam em monitoramento, evitando assim, a aglomeração na unidade de saúde.

Dentre os profissionais de saúde envolvidos no enfrentamento dessa emergência sanitária, encontram-se residentes de Programas de Residência Médica e Multiprofissional em Saúde. Deste modo, intensificando a finalidade de mobilizá-los no exercício laboral, a partir do cenário vigente, o Governo Federal iniciou uma ação estratégica intitulada como "O Brasil Conta Comigo - Residentes na área de Saúde" para o combate à pandemia do novo Coronavírus. Assim, incita-se o propósito de ampliar a cobertura na assistência em todos os níveis de atenção e reduzir o tempo de espera nos atendimentos de usuários do SUS com condições de alto risco em unidades de pronto atendimento e emergências hospitalares nos casos de COVID-19. Em adição, aos profissionais do programa foi concedida bonificação salarial com duração prevista para um período de seis meses ${ }^{6}$.

Com ênfase na atuação do cirurgião-dentista como profissional de saúde da Atenção Primária à Saúde - APS, compreende-se que este atua em ações de promoção e prevenção de doenças não restritas somente à boca, e que podem estar na linha de frente da pandemia, junto aos outros profissionais de saúde. Diante do cenário em vigor, foi orientado pelo Conselho Federal de Odontologia - CFO, em acordo com o protocolo adotado pelo Ministério da Saúde, que fossem suspensos os atendimentos odontológicos eletivos e mantidos apenas procedimentos de urgências e emergência, com o objetivo de diminuir a probabilidade de contaminação cruzada. ${ }^{7,8}$ Tendo vista que o contexto de enfrentamento da COVID-19 estimula mudanças no fazer profissional, provocando atitudes e habilidades que tomam uma dimensão maior, os profissionais acabam por desenvolver novas competências, como a adaptabilidade e a comunicação assertiva.

Diante da potencialidade do CD, enquanto integrante da equipe multiprofissional, que compõe a Estratégia Saúde da Família no combate ao novo coronavírus, assim como, 
na necessidade de reconhecimento deste para além de um cuidado específico com a cavidade oral, verifica-se escassez de estudos qualitativos que avaliem a percepção destes profissionais inseridos em um programa de residência multiprofissional quanto às experiências vividas no campo de prática. ${ }^{9}$ Logo, este relato é importante, haja vista que permite identificar a relevância das reflexões e argumentações do residente, que enquanto cirurgião-dentista, frente à pandemia, atua "além do mocho", através do acolhimento de casos suspeitos, dentre outras atividades laborais. Por conseguinte, desempenhando uma postura ética, responsável e cuidadosa.

A partir do exposto, o objetivo do presente artigo é relatar a atuação de cirurgiõesdentistas, vinculados a um Programa de Residência Multiprofissional em Saúde, em uma Unidade de Saúde da Família, alocada em um município do nordeste brasileiro, no cenário de pandemia relativa à COVID-19.

\section{Metodologia}

Trata-se de um relato de experiência de caráter descritivo e exploratório, da atuação de cirurgiões-dentistas, vinculados ao Programa de Residência Multiprofissional em Saúde da Família da Fundação Estatal Saúde da Família - FESF-SUS em parceria com a Fiocruz, em uma USF, alocada em um município do nordeste brasileiro, durante o enfrentamento da COVID-19, no período entre março e junho de 2020.

Com ênfase na Equipe de Saúde Bucal, os cirurgiões-dentistas cumprem carga horária de 40 horas na Unidade de Saúde, em atividades voltadas ao atendimento odontológico, educação em saúde, visitas domiciliares, reuniões de equipe, dentre outras. Todavia, diante da emergência sanitária vigente, e sob recomendações das normativas governamentais, se fez necessário realizar algumas modificações nas atividades laborais, a fim de adaptar as demandas que surgiram no momento atual. ${ }^{4,10,11}$ Assim, dentre as atuações dessa categoria profissional na USF, incluem-se: acolhimento à demanda espontânea, com realização da estratificação de risco dos usuários; auxílio na resolução das queixas agudas; suporte à equipe médica e de enfermagem nos atendimentos aos pacientes sintomáticos respiratórios; realização de notificação de casos suspeitos de COVID-19; orientação ao usuário na porta de entrada da USF - ação intitulada como "Posso Ajudar", com o intuito de direcionar às demandas que chegam na unidade e orientar/ofertar a higienização das mãos aos usuários antes de adentrar no local; auxílio nas campanhas de vacinação, junto à equipe de enfermagem; e atividades laborais em outros serviços de saúde.

$\mathrm{Na}$ perspectiva de delinear estratégias e encaminhamentos a serem seguidos na prática dessas atividades supracitadas, a equipe da USF formulou um fluxograma de atuação, 
o qual está exposto nesse manuscrito, com o intuito de colaborar com a organização dos serviços e unificar as ações a serem desenvolvidas por todos os profissionais envolvidos.

Assim, este relato foi desenvolvido pela Equipe de residentes do núcleo de Odontologia, alocada na USF referenciada, composta por quatro residentes do primeiro e segundo ano, sob supervisão da preceptoria e apoio pedagógico, vinculados ao núcleo profissional, do referido Programa de residência.

Para subsidiar o aporte teórico da temática abordada, a trajetória metodológica percorrida apoiou-se na análise retrospectiva de estudos/normativas que tiveram enfoque na atuação do cirurgião-dentista, vinculado à APS, frente à COVID-19, contribuindo para o processo de discussão e reflexão das experiências vivenciadas no contexto sanitário vigente.

Para fundamentar a elegibilidade dos estudos referenciados no presente relato, foram considerados como critérios de inclusão: artigos publicados até junho de 2020; estudos primários e secundários que abordaram a temática determinada, disponibilizados na íntegra, e publicados nos idiomas português, inglês ou espanhol. Quanto aos critérios de exclusão, foram definidos: estudos que não abordaram sobre o tema apresentado.

A busca por publicações foi realizada no período entre março e junho de 2020, através das bases de dados eletrônicas Medical Literature and Retrivial System on Line (Pubmed/MEDLINE) e Scientific Eletronic Library Online - SciElo). Ademais, foram utilizados os documentos institucionais disponibilizados pelo Ministério da Saúde, Agência Nacional de Vigilância Sanitária - ANVISA e CFO. Os descritores foram selecionados e, previamente, consultados no Medical Subject Headings - MeSH e no Descritores em Ciências da Saúde DeCS. Após testar os termos isoladamente, identificando grafias alternativas e sinônimos, foram estabelecidos tais descritores: "atenção primária à saúde"; "equipe de assistência ao paciente"; "odontólogos"; "internato e residência"; "infecções por coronavirus".

Por conseguinte, foi realizada a seleção dos estudos através do rastreamento por leitura de títulos e resumos. Após essa etapa, foi feita a leitura na íntegra dos artigos selecionados. Àqueles que obedeceram aos critérios de elegibilidade foram incluídos. Em seguida, executou-se a sistematização das informações e reflexões das experiências adquiridas, desafios enfrentados e sentimentos vivenciados no serviço associado ao cenário da pandemia.

$\mathrm{Na}$ execução deste trabalho, foram atendidas as normas dispostas na Resolução 466/12, do Conselho Nacional de Saúde, ${ }^{12}$ não sendo submetido ao Comitê de Ética em Pesquisa posto que se trata de relato de experiência reflexivo. 


\section{Resultados e Discussão}

ISSN 2179-6750

Compreende-se que a emergência de saúde pública iniciada em Wuhan na China, em dezembro de 2019, relativa ao SARS-CoV-2, se estendeu por todo o mundo. Neste sentido, a partir de março de 2020, a população de um município do nordeste brasileiro, bem como os profissionais de saúde vinculados a uma Unidade de Saúde da Família dessa localidade, vivenciou o enfrentamento a essa, até então, "nova" enfermidade. Como parte dessa equipe, faz-se destaque, nesse relato, a experiência de cirurgiões-dentistas, em processo de qualificação profissional, através da especialização Lato Sensu em Saúde da Família no formato de Residência Multiprofissional, em atuação na Atenção Primária à Saúde.

Esse contexto sanitário fomentou, inicialmente, o despertar de sentimentos frente a esta nova situação jamais vivenciada na história recente. Medo, insegurança e incertezas começaram a fazer parte do cotidiano, à medida que chegavam informações e protocolos a serem seguidos, sob orientação das esferas federal, estadual e municipal. Sentimentos estes que, para cirurgiões-dentistas, se materializaram em afetos anteriormente nunca sentidos, corroborando com experiências relatadas por outros autores. ${ }^{13}$

Para os trabalhadores, a escassez de EPI foi uma das dificuldades encontradas. Durante os atendimentos, a partir dos sinais e sintomas apresentados pelos usuários, notava-se a eminente necessidade de atualização dos saberes apesar das inconstâncias no campo subjetivo afetivo. Ao considerar o que foi observado, percebeu-se que esta situação contribuiu para a manifestação de quadros de cefaleia na maioria dos profissionais ao final do turno de trabalho.

Neste ínterim, o corpo pedagógico do Programa de Residência sugeriu a qualificação dos residentes cirurgiões-dentistas e demais profissionais, a partir da realização de cursos voltados à COVID-19 ofertados por plataformas on-line, como: Universidade Aberta do Sistema Único de Saúde - UNASUS; Conhecimento Livre e Aberto em Saúde - AVASUS; e Fundação Oswaldo Cruz - FIOCRUZ; além da leitura dos Protocolos ${ }^{7}$ e Notas Técnicas ${ }^{5,8}$ do Ministério da Saúde, Agência Nacional de Vigilância Sanitária - ANVISA e CFO. ${ }^{14}$

As novas orientações e protocolos exigiram dos profissionais uma preparação para o enfrentamento da COVID-19, a considerar as características do vírus, diagnóstico clínicoepidemiológico, e vias de transmissão. A partir das normativas supracitadas e a orientação da suspensão dos atendimentos eletivos promovida pelo CFO, o cirurgião-dentista avançou para além das "paredes" do consultório odontológico e se encontrou como profissional de saúde ativo no processo de enfrentamento ao novo coronavírus.

Desta forma, há uma contraposição ao "fazer" centrado no diagnóstico de doenças bucais, $^{15,16}$ e uma conformidade ao modelo de clínica ampliada preconizada pela Política 
Nacional de Humanização - PNH do SUS, a qual preza pelo cuidado integral e holístico, sustentando a ideia de uma atuação clínica do CD focada nas necessidades de saúde da população e que exerce uma abordagem crítica social e reflexiva, humanizada e dialógica, que considera seus sentimentos, valores e saberes. ${ }^{15,16,17,18}$

Para melhor compreensão das adaptações provocadas à atuação da referida categoria dentro do espaço de prática em campo, faz-se necessário expor brevemente o fluxo criado pela equipe da USF a partir deste contexto atípico vivenciado (Figura 1).

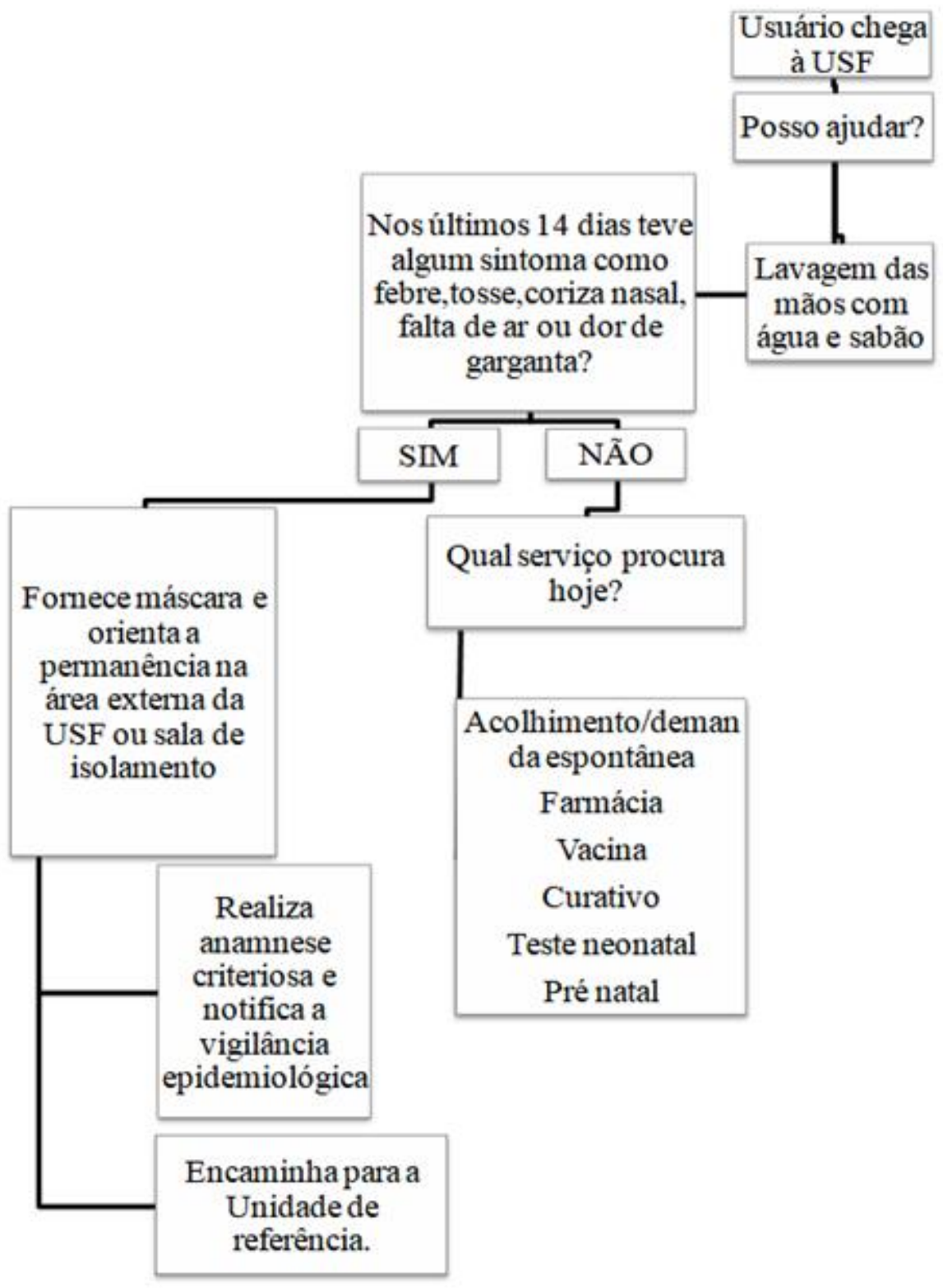

Figura 1. Fluxograma para atendimento na Unidade de Saúde da Família durante a pandemia relativa à COVID-19.

Fonte: Elaborado pelos autores (2020). 
Percebe-se, a partir da análise do fluxograma, que O CD esteve alocado no acolhimento à demanda espontânea, inserindo-se na estratificação de risco dos usuários, auxiliando na resolução das queixas agudas, oferecendo suporte ao núcleo da medicina e enfermagem nos atendimentos aos pacientes sintomáticos respiratórios, bem como na realização de notificação de casos suspeitos de COVID - 19. No momento em que esteve alocado no serviço "Posso ajudar?", o CD orientava o usuário para resolução de sua necessidade, assim como coordenava o fluxo de vacina durante campanha contra a Influenza H1N1, baseando-se na organização dos atendimentos e cumprimento dos critérios exigidos pelo Ministério da Saúde durante as fases da campanha.

Vale ressaltar que o acolhimento à demanda espontânea dentro da USF até março de 2020 era composto por três profissionais de nível superior: médico, enfermeiro, cirurgiãodentista e/ou profissional do NASF-AB. Estes se organizavam entre primeira escuta, pautada na abordagem inicial durante chegada do usuário à unidade, e segunda escuta, marcada pela necessidade de resolutividade da demanda de saúde trazida. Após a pandemia, houve redução de um profissional para realocá-lo em outras atividades. Desta forma, o cirurgiãodentista ou profissional que compõe o NASF-AB passaram a ocupar necessariamente a primeira escuta, estando o médico ou enfermeiro na segunda escuta do acolhimento.

Destaca-se, assim, que o Acolhimento à Demanda Espontânea abrange um espaço potente de troca com os usuários e aprendizado mútuo. Compreende-se que este lugar favorece o desenvolvimento de competências que inicialmente vinculam-se às categorias de medicina e enfermagem, incluindo renovação de receita e diagnóstico de doenças sistêmicas, como Esporotricose, infecção fúngica apresentada como micose subcutânea, subaguda ou crônica de transmissão zoonótica principalmente por gatos infectados $^{19,20}$. Através deste relato de experiências, não se pretende mudar o foco de atuação da referida categoria, mas enfatizar este espaço potente de construção de saberes.

À vista disso, a participação do cirurgião-dentista no acolhimento à demanda espontânea e no "Posso ajudar?" não foi tarefa difícil, mesmo que distante das "paredes" do consultório odontológico, visto que, antes da pandemia, já se cumpria esta atuação. Salienta-se que a abertura para participação da odontologia nestes lugares não foi enrijecida pelas outras categorias profissionais atuantes na USF, tanto de nível superior quanto técnico. Acrescenta-se o fato de que a cadeira odontológica estava impossibilitada de uso em detrimento do problema mecânico do compressor desde dezembro de 2019.

Este destaque ao "novo fazer" da Odontologia na Atenção Primária à Saúde, despertou discussão dentro da equipe de saúde bucal quanto à rigidez da formação acadêmica pautada pelo curso de Odontologia a qual reforça as ciências biomédicas, atenção centrada no diagnóstico de doenças e atuação no setor privado. Esta formação, por 
vezes, pode impactar negativamente no processo de trabalho dentro da ESF e dificultar a abertura para troca de conhecimentos e saberes com demais membros da equipe. ${ }^{15-17,21-23}$

É de fácil percepção que a formação ofertada pelas Instituições de Ensino Superior caracteriza-se por preparar profissionais de saúde focados em intervenções em âmbito clínico restrito pelas competências específicas, de forma fragmentada e individualizada. Diante do contexto da ESF e de políticas que preconizam um atendimento humanizado no SUS, os profissionais de saúde acabam por reformular o seu fazer, adquirindo competências a nível multiprofissional, como o enfoque na humanização da atenção, acolhimento, vínculo e trabalho em equipe. Sabe-se que o $C D$, particularmente, tem uma formação voltada basicamente para a prática clínica e muitos desafios dentro do contexto exigido para o ofício na ESF são esperados para estes profissionais ${ }^{18}$. Dessa forma, o fazer dos cirurgiõesdentistas, diante da presente conjuntura, reestruturou-se, de modo que, hábitos e atuações adquiridas na formação superior tradicional fossem agregados a atitudes preconizadas por uma formação em saúde voltada para a construção de competências em comum com os outros profissionais e colaborativas, sem deixar de lado as especificidades da área.

Estudos mostram que aos discentes de Odontologia, em sua maioria, não são ensinados métodos de escuta e acolhimento, bem como experiências para criação de vínculo com o usuário, e sim, é posto o cuidado diretamente relacionado à oferta de tecnologias duras e não se consideram diversos outros fatores que são imprescindíveis à prática odontológica. O que pode ser justificado pelo fato da construção de saberes nos cursos de graduação de Odontologia ainda ocorre através de disciplinas fragmentadas, cuja formação não está focada no cuidado ao usuário e seus desejos. Dessa forma, torna-se um desafio para o profissional CD ofertar atenção integral humanizada, realizar trabalho em equipe e compreender a realidade em que vive a população. ${ }^{15,16,24}$

A partir desse entrave, observa-se a importância da fundamentação do cirurgiãodentista em tecnologias do cuidado em saúde para o seu exercício laboral. Em verdade, são nos processos micropolíticos construídos diariamente, traduzidos pela relação estabelecida entre profissionais de saúde e indivíduos/comunidade, que se pode perceber o emprego das tecnologias do cuidado em saúde. Estas abrangem as técnicas, procedimentos e conhecimentos utilizados na resolução das demandas despontadas para o cuidado. Existem três tipos de tecnologias empregadas: leves, leve-duras e duras. As leves, essenciais no processo de acolhimento do indivíduo/coletividade, configuram-se como tecnologias de relação, escuta, interesse, vínculo e autonomia, constituída por um trabalho vivo e que está acima do campo das práticas de saúde. Enquanto as duras utilizam os equipamentos tecnológicos, manuseio de instrumentais, utilização de recursos materiais para raciocínio 
lógico e procedimentos clínicos. Já as leve-duras englobam a interação com o usuário, junto aos procedimentos clínicos, ou seja, a junção das duas anteriores. ${ }^{18,25}$

Assim, com a reformulação do pensamento crítico e o fazer baseado em princípios do SUS como integralidade, equidade e universalidade, o atendimento prestado aos pacientes tende a afastar o enfoque clínico-curativista (predomínio da tecnologia leve-dura e dura), e aproxima a tecnologia leve, ofertando acolhimento a esse usuário, trazendo como prioridade a escuta qualificada, o entendimento do ser biopsicossocial, envolvido por uma cadeia de relações externas e que influenciam no processo saúde-doença apresentado.

Dessa forma, torna-se eminente que instituições de ensino superior do curso de odontologia promovam estratégias que fomentem à prática efetiva de tecnologias leves, ou seja, estimulem o exercício da odontologia para além das tecnologias leve-duras e duras; que integrem ensino - serviço e trabalho em equipe na saúde, ao ofertar, durante a graduação e pós graduação, espaços de interação entre profissionais de diferentes áreas da saúde para elaboração de planejamentos terapêuticos em conjunto, a fim de formar profissionais voltados para o trabalho colaborativo em equipe interprofissional, mais seguros e garantidores da qualidade do cuidado em saúde. ${ }^{26}$

Neste sentido, compreendendo que Programas de Residência Multiprofissional em saúde permite ao profissional a participação em espaços com atuação interdisciplinar, com vistas à atenção integral humanizada, percebe-se o quão é primordial a inserção do cirurgião-dentista, principalmente àquele atuante na APS, nessa experiência profissional. Fato que reforça a importância desse tipo de interação para construção de novos saberes e renovação do fazer em saúde, destacando-se a qualificação da prática e participação ativa do aprendiz através da problematização vivenciada em campo.

Sabe-se que a integralidade da atenção à saúde é fundamental para ofertar aos usuários do SUS um cuidado ampliado e eficaz, tendo em vista a necessidade de considerálo como sujeito inserido em um contexto social, familiar e cultural que carrega intensa subjetividade. $^{27,28}$ Assim, estratégias dialógicas que pautam atividades de educação em saúde são fundamentais para mudança de hábitos. Dentro do atual cenário da pandemia observou-se redução do número de queixas odontológicas no acolhimento à demanda espontânea da referida Unidade de Saúde da Família. Fato que gerou alguns questionamentos para a equipe de saúde bucal: "Será que as estratégias de educação em saúde utilizadas pela equipe à longo prazo têm reverberado na redução de casos de urgência odontológica?" ou "Sinais e sintomas gerados a partir de situações álgicas, provocadas por patologias bucais, são consideradas segundo plano no cuidado em saúde das pessoas que residem no território adscrito?" ou ainda "A ciência de que o compressor odontológico não funciona há meses, torna a equipe de saúde incapaz de gerar cuidado 
fora da cadeira odontológica?". Estas representam dúvidas que podem gerar pesquisa posterior.

Neste ínterim, a necessidade de despontar ações de educação e promoção da saúde necessárias ao profissional inserido na $\mathrm{ESF}^{10}$ durante o contexto de combate à COVID-19, mobilizou a equipe multiprofissional na produção de cartazes informativos, instrução sobre as formas de prevenção através do uso adequado de máscaras de pano, lavagem e desinfecção das mãos, assim como a necessidade de manter o isolamento social e procura da USF em caso de sintomas respiratórios.

Diante do exposto, observa-se que a busca por novos caminhos e o desbravar de novas habilidades, em meio ao enfrentamento da COVID-19, possibilitou a ampliação de conhecimentos e aprimoramento de competências existentes. Logo, intensificou-se a capacidade de superar as adversidades e adaptar-se às mudanças, afinal se fez necessário inúmeras reavaliações diárias de estratégias a serem seguidas pela equipe, a fim de viabilizar de forma coletiva a assertividade as ações de cuidado prestadas à população, o que denotam a importância, também, de outras habilidades essenciais, como a empatia, a flexibilidade e o trabalho em equipe.

\section{Considerações finais}

Esta experiência trouxe à tona a potencialidade do cirurgião-dentista enquanto profissional de saúde, dotado de capacidades para além dos cuidados à cavidade bucal, o qual pode aplicar e aprimorar competências, a fim de promover resolutividade às demandas trazidas pelos usuários, além de fomentar encaminhamentos inerentes aos pontos da rede de atenção em saúde.

Por outro lado, é válido ressaltar que a partir do relato exposto, identifica-se que o cirurgião-dentista, integrante da Equipe de Saúde da Família, possui habilidades para, junto às outras categorias, comporem a linha de frente nesse processo de enfrentamento à COVID-19. Destarte, a ciência está em incessante movimento e a Odontologia deve seguir a mesma jornada. Afinal, ir além do sorriso não significa ir atrás de tendências, e sim ir ao rumo da evidência, adaptabilidade, comunicação assertiva e cuidado em saúde para além da boca.

\section{Referências}

1. AVASUS: Conhecimento livre e aberto [internet]. Vírus respiratórios emergentes, incluindo a COVID-19 [citado 15 abr. 2020]. Disponível em: https://avasus.ufrn.br/local/avasplugin/cursos/curso.php?id=320. 
2. Organização Mundial da Saúde [internet]. Fluxo de manejo clínico na Atenção Primária à saúde em transmissão comunitária [acesso em 15 de abril de 2020]. Disponível em: https://www.conasems.org.br/covid-19-protocolos-e-orientacoes-aos-profissionais-eservicos-de-saude/.

3. Secretaria de Atenção Primária à Saúde (SAPS) (Brasil)[internet]. Atendimento odontológico no SUS. 2020. [citado 15 de abr. 2020]. Disponível em: http://website.cfo.org.br/wp-content/uploads/2020/03/COVID-19_ATENDIMENTOODONTOLOGICO-NO-SUS.pdf.

4. Ministério da Saúde (BR). Universidade Aberta do SUS. Atualização: orientações gerais ao paciente com COVID-19 na Atenção Primária à Saúde [internet]. 2020 [citado 15 abr. 2020]. Brasília, DF: MS. Disponível em: https://www.unasus.gov.br/cursos/curso/46168.

5. Agência Nacional de Vigilância Sanitária (BR). Nota técnica n. 04, de 30 de janeiro de 2020. Orientações para serviços de saúde: medidas de prevenção e controle que devem ser adotadas durante a assistência aos casos suspeitos ou confirmados de infecção pelo novo coronavírus (sars-cov-2).

6. Ministério da Saúde (BR). Portaria n. 580, 27 de março de 2020. Dispõe sobre a Ação Estratégica "O Brasil Conta Comigo - Residentes na área de Saúde", para o enfrentamento à pandemia do coronavírus (COVID-19). Brasília, DF: Diário Oficial da União; 30 de março de 2020.

7. Secretaria de Atenção Primária à Saúde (BR). Protocolo de manejo clínico do coronavírus (Covid-19) na atenção primária à saúde. 2020 [citado 15 abr. 2020]. Disponível em: https://www.saude.gov.br/images/pdf/2020/marco/20/20200318ProtocoloManejover002.pdf. Acesso em 17 de maio de 2020.

8. Ministério da Saúde (BR). Nota Técnica No 9/2020-CGSB/DESF/SAPS/MS. COVID-19 e atendimento odontológico no SUS [internet]. 2020 [citado 17 maio 2020]. Disponível em: http://189.28.128.100/dab/docs/portaldab/documentos/COVID_19_ATENDIMENTO\%20OD ONTOLOGICO_SUS_APS_20200319_ver001.pdf.

9. Franco AG, Amorim JC, Carvalho GAP, Dias SC, Franco ABG. Importância da conduta do cirurgião-dentista frente à contenção e prevenção do Covid-19. Inter American J Med Health. 2020 abr;3: e202003011. https://doi.org/10.31005/iajmh.v3i0.86. 
10. Pereira $M L$, Azevedo $A$. Da emergência de um novo vírus humano à disseminação global de uma nova doença: A COVID -19 - os desafios para a saúde oral [internet]. [s.d.] [citado 15 abr. 2020]. Disponível em:

http://asset.youoncdn.com/ab296ab30c207ac641882479782c6c34/98c48d4df93f8ffcc282e 7ec035216ed.pdf.

11. Comitê de Odontologia AMIB/CFO de enfrentamento ao COVID-19. Recomendações AMIB/CFO para atendimento odontológico COVID - 19. Departamento de Odontologia AMIB - $1^{\circ}$ Atualização. Departamento de Odontologia AMIB-1 Atualização; março 2020.

12. Conselho Nacional de Saúde (BR). Resolução n. 466, de 12 de dezembro de 2012. Aprova as diretrizes e normas regulamentadoras de pesquisas envolvendo seres humanos. Brasília, DF: Ministério da Saúde; 2012.

13. Lucas AB, Freitas NA, Freitas FD, Ponte Neto AO, Lacerda RA, Cavalcante VOM. Sentimentos que transpõem a residência multiprofissional em saúde da família: relato de experiência. Sanare (Sobral). 2016;15(2):154-9.

14. Conselho Federal de Odontologia (BR). Resolução n. 226, de 04 de junho de 2020. Dispõe sobre o exercício da Odontologia à distância, mediado por tecnologias, e dá outras providências. Brasília, DF: CFO; 2020.

15. Bastos BRMS, Serra Clara A, Fonsêca GS, Pires FS, Souza CR, Botazzo C. Formação em saúde bucal e clínica ampliada: por uma discussão dos currículos de graduação. Rev ABENO. 2017;17(4):73-86. https://doi.org/10.30979/rev.abeno.v17i4.520.

16. Graff VA, Toassi RFC. Produção do cuidado em saúde com foco na Clínica Ampliada: um debate necessário na formação em Odontologia. Rev ABENO. 2017;17(4):63-72. http://dx.doi.org/10.30979/rev.abeno.v17i4.516.

17. Hayacibara MF, Lolli LF, Terada RSS, Hidalgo MM, Bispo CGC, Terada HH, et al. Experiência de Clínica Ampliada em Odontologia na Universidade Estadual de Maringá. Rev Bras Edu Med. 2012;36(1Supl.2):178-83. https://doi.org/10.1590/S0100-55022012000300026.

18. Moretti-Pires RO, Bueno SMV. Freire e formação para o Sistema Único de Saúde: o enfermeiro, o médico e o odontólogo. Acta Paul Enferm. 2009;22(4):439-44. https://doi.org/10.1590/S0103-21002009000400015.

19. Bazzi T, Melo SMP, Fighera RA, Kommers GD. Características clínico-epidemiológicas, histomorfológicas e histoquímicas da esporotricose felina. Pesq Vet Bras. 2016;36(4):30311. https://doi.org/10.1590/S0100-736X2016000400009. 
20. Reis WG, Scherer MDA, Carcereri DL. O trabalho do Cirurgião-Dentista na Atenção Primária à Saúde: entre o prescrito e o real. Saude Debate. 2015;39(104):56-64. https://doi.org/10.1590/0103-110420151040608.

21. Rodrigues AAAO, Fonsêca GS, Siqueira DVS, Assis MMA, Nascimento MAA. Práticas da equipe de saúde bucal na estratégia saúde da família e a construção (des)construção da integralidade em Feira de Santana-BA. Rev APS. 2010;13(4):476-85.

22. Xavier GM. A formação do cirurgião-dentista no contexto do sistema único de saúde: uma avaliação do ensino de odontologia [dissertação]. [Brasília, DF]. Universidade de Brasília; 2013. Disponível em: https://repositorio.unb.br/handle/10482/14868.

23. Araújo YP, Dimenstein M. Estrutura e organização do trabalho do cirurgião-dentista no PSF de municípios do Rio Grande do Norte. Cienc Saude Colet. 2006;11(1);219-27. http://dx.doi.org/10.1590/S1413-81232006000100031.

24. Uchôa PA, Vasconcelos MVL. Percepção dos discentes sobre as práticas colaborativas em um estágio integrado em saúde. CIAIQ2018. 2018[citado 25 jun. 2020];1(Atas Investigação Qualitativa em Educação). Disponível em: https://proceedings.ciaq.org/index.php/ciaiq2018/article/view/1649.

25. Merhy EE, Baduy RS, Seixas CT, Almeida DES, Slomp Júnior H. Avaliação compartilhada do cuidado em saúde: surpreendendo o instituto nas redes. 1a ed. Rio de Janeiro: Hexis. 2016. 448 p. (vol. 1).

26. Silva JAM, Peduzzi M, Orchard C, Leonello VM. Educação interprofissional e prática colaborativa na Atenção Primária à Saúde. Rev Esc Enferm USP. 2015;49(n. spec 2):16-24. http://dx.doi.org/10.1590/S0080-623420150000800003.

27. Farias MR, Sampaio JJC. Papel do cirurgião-dentista na equipe de saúde da família. RGO Rev Gauch Odontol. 2011;59(1):109-15.

28. Souza MC, Araújo TM, Reis Júnior WM, Souza JN, Vilela ABA, Franco TB. Integralidade na atenção à saúde: um olhar da Equipe de Saúde da Família sobre a fisioterapia. Mundo Saude (São Paulo). 2012;36(3):452-60. 


\section{Minicurrículo}

\section{Jamille Souza Xavier dos Santos | ORCiD: 0000-0002-7123-3384}

Cirurgiã-dentista residente vinculada ao Programa de Residência Multiprofissional em Saúde da Família pela Fundação Estatal Saúde da Família FESF-SUS em parceria com a Fiocruz Bahia, Camaçari, BA.

Andresa Santos Silva | ORCiD: 0000-0003-0122-5917

Cirurgiã-dentista residente vinculada ao Programa de Residência Multiprofissional em Saúde da Família pela Fundação Estatal Saúde da Família FESF-SUS em parceria com a Fiocruz Bahia, Camaçari, BA.

Leilane dos Anjos de Carvalho | ORCiD: 0000-0002-2911-2335

Cirurgiã-dentista residente vinculada ao Programa de Residência Multiprofissional em Saúde da Família pela Fundação Estatal Saúde da Família FESF-SUS em parceria com a Fiocruz Bahia, Camaçari, BA.

\section{Juliana Oliveira Soares | ORCiD: 0000-00103-0385-9438}

Cirurgiã-dentista residente vinculada ao Programa de Residência Multiprofissional em Saúde da Família pela Fundação Estatal Saúde da Família FESF-SUS em parceria com a Fiocruz Bahia, Camaçari, BA.

\section{Síntique Priscila Alves Lopes | ORCiD: 0000-00011-5750-5614}

Cirurgiã-dentista mestranda em Odontologia e Saúde pela Universidade Federal da Bahia-UFBA, preceptora do Programa de Residência Multiprofissional em Saúde da Família pela Fundação Estatal Saúde da Família FESF-SUS em parceria com a Fiocruz Bahia, Camaçari, BA.

Marcela Beatriz Aguiar Moreira | ORCiD: 0000-0002-2545-2121

Cirurgiã-dentista mestre e doutoranda em Saúde Coletiva pela Universidade Estadual de Feira de Santana UEFS, apoiadora pedagógica do núcleo de odontologia do Programa de Residência Multiprofissional em Saúde da Família pela Fundação Estatal Saúde da Família FESF-SUS em parceria com a Fiocruz Bahia, Camaçari, BA. 